\title{
Chronic cerebrospinal venous insufficiency is not associated with cognitive impairment in multiple sclerosis
}

\author{
Ralph HB Benedict ${ }^{1,3^{*}}$, Bianca Weinstock-Guttmam ${ }^{1}$, Karen Marr ${ }^{2}$, Vesela Valnarov ${ }^{2}$, Cheryl Kennedy ${ }^{2}$, Ellen Carl ${ }^{2}$, \\ Christina Brooks' ${ }^{2}$ David Hojnacki ${ }^{1}$ and Robert Zivadinov ${ }^{1,2}$
}

\begin{abstract}
Background: Chronic cerebrospinal venous insufficiency (CCSVI) has been reported in multiple sclerosis (MS) yet its significance in relation to cognitive function is undetermined.

This study measured the association between the presence and severity of CCSVI and cognitive impairment in patients with MS.

Methods: CCSVI was assessed using extra-cranial and trans-cranial Doppler sonography in 109 MS patients (79 with relapsing-remitting, 23 with secondary-progressive and 7 with primary-progressive disease subtype). A subject was considered CCSVI-positive if $\geq 2$ venous hemodynamic criteria were fulfilled. The Minimal Assessment of Cognitive Function in MS (MACFIMS) battery was administered assessing the full spectrum of cognitive domains known to be affected by MS. Depression was quantified using the Beck Depression Inventory Fast Screen (BDIFS). Partial correlations, analysis of variance (or covariance) and linear regression were used to examine the hypothesis that CCSVI status is related to cognition or depression after controlling for education and gender.

Results: There were 64 (58.7\%) patients who were considered CCSVI-positive. The regression models predicting venous hemodynamic insufficiency severity score were not statistically significant for any of the MACFIMS predictor variables. The analysis of variance tests showed a significant effect of CCSVI-positive diagnosis on cognitive ability in only one of the 10 MACFIMS outcomes, and that one was in the opposite direction of the tested hypothesis. There was no correspondence between CCSVI diagnosis and depression, as measured by the BDIFS.
\end{abstract}

Conclusions: We find no evidence of an association between the presence and severity of CCSVI with cognitive impairment and depression in patients with MS.

Keywords: Multiple sclerosis, CCSVI, Cognition

\section{Background}

Multiple sclerosis (MS) is an inflammatory disease of the central nervous system, causing both demyelination and neurodegeneration [1,2]. As would be expected, a substantial number, roughly 50\% [3-5], of MS patients have cognitive impairment. In recently diagnosed or benign course patients, the incidence ranges from $20 \%$ to $40 \%$ $[5,6]$ whereas in samples with a substantial secondary

\footnotetext{
* Correspondence: benedict@buffalo.edu

'Department of Neurology, State University of New York at Buffalo, 100 High St, Buffalo, NY 14203, USA

${ }^{3}$ Department of Neurology, School of Medicine and Biomedical Sciences, 100 High St, Buffalo, NY 14203, USA

Full list of author information is available at the end of the article
}

progressive course, roughly $60 \%$ of patients are affected [4]. The correlation between cognitive impairment and brain atrophy is robust [7-9]. However, why some patients show cognitive impairment and brain atrophy while others do not is poorly understood.

Chronic cerebrospinal venous insufficiency (CCSVI) was first reported in MS patients in 2009 [10]. As a vascular condition, CCSVI is characterized by anomalies of the main extra-cranial cerebrospinal venous routes, mainly in internal jugular and azygos veins that are hypothesized to interfere with normal venous outflow from the brain to the periphery. Since then, the topic has met with unprecedented controversy following a wide range of reported

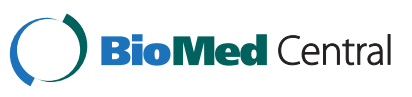


CCSVI frequencies in MS studies [11-13]. Diagnosis of CCSVI implies a pathological condition the determination of which is based mainly on color Doppler sonography (DS) of extra- (neck) and intra-cranial veins using five venous hemodynamic $(\mathrm{VH})$ criteria (with cutoff of $\geq 2$ positive criteria used for a diagnosis of CCSVI) $[10,14]$. So far, published studies comparing the prevalence of CCSVI in MS patients and controls [12,15] have not reproduced the original findings of Zamboni et al. showing 100\% sensitivity/specificity $[10,14]$. While some groups did report a higher prevalence in MS patients than controls $[16,17]$, others reported the opposite, that is, no greater frequency in MS than in healthy persons [16,18-22]. In the largest cohort studied to date, we found a CCSVI frequency of $56.1 \%$ in MS patients compared to $22.7 \%$ in healthy controls [23]; however, the condition was also detected at a high frequency in patients with other neurologic diseases.

While not causative, some studies suggest that CCSVI may be a risk factor for clinical worsening in MS [24-26], although here, too, there are contradictory results $[16,20]$. In a large cohort study exploring the association between CCSVI status and both lesion burden and brain atrophy in MS, no relationship was found [27].

If CCSVI is a risk factor for neurodegeneration or progressive neurologic disability, we would expect significant correlation between CCSVI and cognitive impairment within MS cohorts. The present study was intended to examine this hypothesis.

\section{Methods}

\section{Participants}

The neuropsychological data were collected in a singlecenter, cross-sectional rater-blinded study that included patients with definite MS who were undergoing determination of CCSVI status. Exclusion criteria were as follows: (a) presence of relapse or steroid treatment in the 30 days preceding study entry; (b) pre-existing medical conditions known to be associated with brain pathology; (c) pre-existing neuropsychiatric conditions known to be associated with cognitive impairment, including, for example, learning disability, major depressive disorder, schizophrenia and traumatic brain injury, among others; (d) history of cerebral congenital vascular malformations; (e) current alcohol or drug abuse; and (f) pregnancy. Participants underwent a clinical and neuropsychological examination, as well as both trans- and extra-cranial DS. Demographic and clinical information on all participating subjects was acquired using a structured questionnaire and by examination. The collected data included age, sex, age at disease onset, age at diagnosis, symptoms at disease onset and diagnosis, disease duration, Expanded Disability Status Scale (EDSS) [28], disease subtype [29] and the results of physical examination.
The study was approved by the Institutional Review Board and informed consent was obtained from all patients.

\section{Neuropsychological assessment}

The neuropsychological examination was performed by trained personnel who were blinded to the subjects' clinical and CCSVI characteristics. While patients with current major depressive episode were excluded from the study, remitted or minor depression was permitted, and the degree was quantified using the Beck Depression Inventory Fast Screen (BDIFS) [30] which has been validated in MS [31].

Next, the Minimal Assessment of Cognitive Function in MS (MACFIMS) battery was administered [32], assessing the full spectrum of cognitive domains known to be affected by MS. The MACFIMS has been tested using large prospective MS samples [4,33] and its psychometric properties have been established through the development of the individual tests and further research on the overall battery [34]. Also, the tests on the MACFIMS correlate well with brain magnetic resonance imaging (MRI) metrics in MS samples [35,36]. The specific tests included are as follows: the oral response - version of the Symbol Digit Modalities Test (SDMT) [37], the Paced Auditory Serial Addition Test (PASAT) [38], the California Verbal Learning Test, 2nd edition (CVLT2) [39], the Brief Visual Memory Test, Revised (BVMTR) [40], the Controlled Oral Word Association Test (COWAT) [41], the Judgment of Line Orientation Test (JLO) [42] and the Delis-Kaplan Executive Function System (DKEFS) Sorting Test [43]. The tests were normalized on the basis of recently published normative data that account for demographics, such as age and education [33].

\section{Doppler sonography}

Extra-and trans-cranial DS was performed on a color-coded DS scanner (MyLab 25; Esaote-Biosound, Irvine, CA, USA) equipped with a 5.0- to $10-\mathrm{Mhz}$ transducer to examine venous return in the internal jugular veins (IJVs) and venous veins (VVs). The DS examination was performed by two trained technologists who were blinded to the subjects' demographic, clinical and neuropsychological characteristics. The detailed scanning protocol and validation were previously reported [23]. Briefly, the following five $\mathrm{VH}$ parameters indicative of CCSVI were investigated: 1) reflux/bidirectional flow in the IJV and/or in the VV in sitting and in supine positions, defined as flow directed towards the brain for a duration of $>0.88$ second; 2) reflux/bidirectional flow in the deep cerebral veins defined as reverse flow for a duration of 0.5 second in one of the intra-cranial veins; 3) B-mode abnormalities or stenoses in IJVs, defined as a cross-sectional area (CSA) of this vein $\leq 0.3 \mathrm{~cm}^{2}$; 4) flow that is not Doppler-detectable in IJVs and/or VVs despite multiple deep breaths; and 5) reverted 
postural control of the main cerebral venous outflow pathway by measuring the difference of the CSA of the IJVs in the supine and upright positions. A subject was considered CCSVI-positive if $\geq 2 \mathrm{VH}$ criteria were fulfilled, as previously proposed [10].

We calculated the $\mathrm{VH}$ insufficiency severity score (VHISS) [14,44], defined as a weighted sum of the scores contributed by each individual $\mathrm{VH}$ criterion. The formula for the VHISS calculations is: VHISS = VHISS1 + VHISS2 + VHISS3 + VHISS4 + VHISS5. The VHISS score is an ordinal measure of the overall extent and number of $\mathrm{VH}$ flow pattern anomalies, with a higher value of VHISS indicating a greater severity of abnormal flow. The minimum possible VHISS value is 0 and the maximum 16 .

\section{Statistical analyses}

Statistical analyses were performed using SPSS software. As noted above, for descriptive purposes, the raw test scores derived from neuropsychological examination were normalized using previously published data [33]. Partial correlations were performed using the Pearson productmoment correlation coefficient, and the CCSVI positive and negative groups were compared using analysis of variance (or covariance) and chi-square tests. Linear regression was used to examine the hypothesis that CCSVI status as measured by the VHISS score is related to cognitive function or depression, after controlling for education and gender. Throughout, we employed a conservative threshold of $\mathrm{P}<0.01$ to control for type 1 error.

\section{Results}

Of the 109 patients enrolled, 79 were diagnosed with relapsing-remitting, 23 with secondary-progressive, and 7 with primary-progressive disease subtype. All were Caucasian, except two African-Americans and two of Latin American heritage. The other descriptive statistics including demographic, clinical, depression and cognitive outcomes are presented in Table 1. The CCSVI positive and negative MS groups were well matched, and no age, disease duration, EDSS or disease subtype differences were found. While the pattern of cognitive impairment was the same as described in previous studies (SDMT and BVMTR most sensitive), overall we found less impairment in this sample as compared to some previous studies using the same test battery [7,45-47].

There were $64(58.7 \%)$ patients considered CCSVIpositive and 45 negative (Table 1). As shown in Figure 1, the total criteria VHISS score ranged 0 to 8 . The median was represented by 26 patients achieving a score of 3 .

There were modest trends toward linear correlation between education $(r=0.25)$ and gender $(r=-0.15)$ and the VHISS score. The chi-square test showed correspondence

Table 1 Demographic, clinical and neuropsychological characteristics in multiple sclerosis patients with positive and negative diagnosis of chronic cerebrospinal venous insufficiency (CCSVI)

\begin{tabular}{|c|c|c|c|c|c|c|c|c|c|}
\hline & \multicolumn{5}{|c|}{ All patients } & \multicolumn{2}{|c|}{$\begin{array}{l}\text { CCSVI positive } \\
\text { number }=64\end{array}$} & \multicolumn{2}{|c|}{$\begin{array}{c}\text { CCSVI negative } \\
\text { number }=45\end{array}$} \\
\hline & Median & Mean & SD & Range & Mean z & Mean & SD & Mean & SD \\
\hline Age & 48 & 47.3 & 9.8 & $25-66$ & - & 46.8 & 9.0 & 47.6 & 10.4 \\
\hline Education & 16 & 16.0 & 2.3 & $12-20$ & - & 15.8 & 2.3 & 16.1 & 2.3 \\
\hline Male/female & \multicolumn{3}{|c|}{$38 / 72$} & & - & \multicolumn{2}{|c|}{$27 / 38$} & \multicolumn{2}{|c|}{$11 / 34$} \\
\hline Disease duration in years & 9 & 10.5 & 8.2 & $01-41$ & - & 10.6 & 8.5 & 10.5 & 8.1 \\
\hline Disease course: RR/SP/PP & \multicolumn{3}{|c|}{$79 / 23 / 07$} & & - & \multicolumn{2}{|c|}{$48 / 13 / 03$} & \multicolumn{2}{|c|}{$31 / 10 / 04$} \\
\hline EDSS & 2.5 & 3.3 & 1.9 & $0-7.5$ & - & 3.2 & 1.9 & 3.5 & 2.0 \\
\hline Beck Depression Inventory Fast Screen & 1 & 2.4 & 2.6 & $0-16$ & - & 2.5 & 2.8 & 2.4 & 2.4 \\
\hline Symbol Digit Modalities Test & 53 & 53.5 & 11.7 & $9-74$ & -0.62 & 54.9 & 12.0 & 51.5 & 11.3 \\
\hline Paced Auditory Serial Addition Test & 48 & 44.3 & 13.8 & $0-60$ & -0.41 & 45.5 & 12.6 & 42.7 & 15.6 \\
\hline CVLT2Total Learning & 55 & 54.0 & 11.1 & $23-87$ & 0.37 & 55.3 & 12.4 & 52.1 & 8.6 \\
\hline CVLT2Delayed Recall & 12 & 11.5 & 3.2 & $2-16$ & 0.17 & 11.7 & 3.5 & 11.1 & 2.7 \\
\hline BVMTR Total Learning & 23 & 22.1 & 6.7 & $4-34$ & -0.32 & 22.9 & 6.8 & 20.9 & 6.6 \\
\hline BVMTR Delayed Recall & 9 & 8.6 & 2.4 & $1-12$ & -0.63 & 9.0 & 2.3 & 7.9 & 2.5 \\
\hline Controlled Oral Word Association Test & 37 & 39.1 & 10.9 & $14-64$ & 0.26 & 39.6 & 11.1 & 38.0 & 10.7 \\
\hline Judgment of Line Orientation Test & 26 & 24.9 & 3.9 & $12-30$ & -0.59 & 25.6 & 3.4 & 24.0 & 4.6 \\
\hline DKEFS Sorting Correct Sorts & 11 & 10.7 & 2.2 & $3-15$ & -0.07 & 10.7 & 2.3 & 10.7 & 2.0 \\
\hline DKEFS Sorting Description Score & 40 & 40.3 & 8.7 & $10-58$ & -0.04 & 40.4 & 9.2 & 40.1 & 7.9 \\
\hline
\end{tabular}




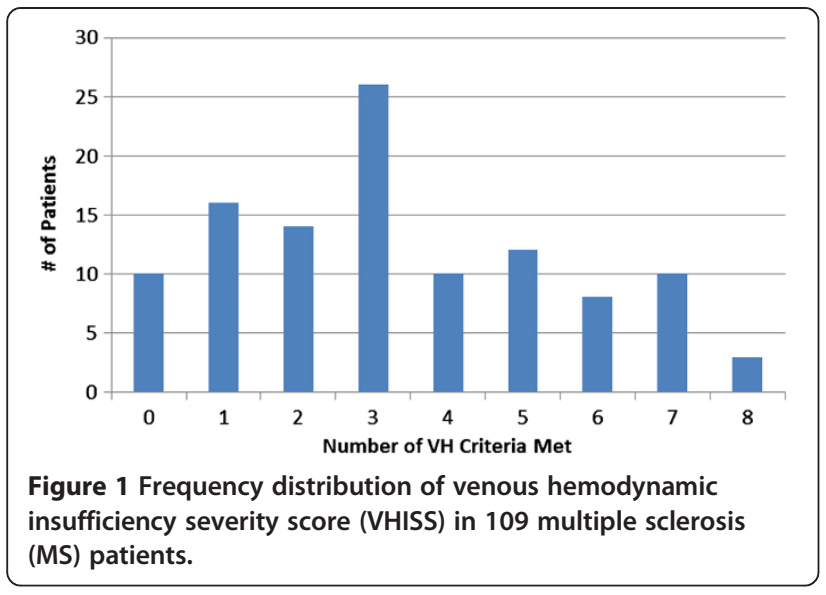

between gender and the CCSVI-positive diagnosis $(P=0.05)$. Therefore, education and gender were controlled for in hypothesis testing models. There was no correspondence between CCSVI and depression as measured by the BDIFS.

The regression models predicting VHISS score after controlling for education and gender were not statistically significant for any of the MACFIMS predictor variables. The largest partial $r$ in the analysis was -0.13 between CVLTR Delayed Recall and the VHISS score (Table 2).

The analysis of covariance (ANCOVA) tests showed a significant effect of CCSVI-positive diagnosis on cognitive ability in one of the 10 MACFIMS outcomes (Table 1, Figure 2). For BVMTR Delayed Recall, CCSVIpositive patients showed better performance than their CCSVI-negative counterparts $(P=0.009)$. The direction of the effect was thus counter to expectation in that positive patients achieved a score of 9.1 compared to 7.8 for the CCSVI negative group.

Table 2 Correlation coefficients between venous hemodynamic insufficiency severity score (VHISS) and depression and cognition scores in multiple sclerosis patients $(n=109)$

\begin{tabular}{lcc}
\hline & Zero order $\mathbf{r}$ & Partial $\mathbf{r}$ \\
\hline Beck Depression Inventory Fast Screen & 0.10 & 0.13 \\
Symbol Digit Modalities Test & -0.03 & -0.02 \\
Paced Auditory Serial Addition Test & -0.04 & -0.07 \\
CVLT2Total Learning & -0.06 & -0.04 \\
CVLT2Delayed Recall & -0.13 & -0.13 \\
BVMTR Total Learning & 0.06 & 0.04 \\
BVMTR Delayed Recall & 0.10 & 0.09 \\
Controlled Oral Word Association Test & 0.02 & -0.03 \\
Judgment of Line Orientation Test & 0.13 & 0.00 \\
DKEFS Sorting Correct Sorts & 0.04 & -0.05 \\
DKEFS Sorting Description Score & 0.08 & -0.01 \\
\hline
\end{tabular}

BVMTR Brief Visuospatial Memory Test, Revised, CVLT2 California Verbal Learning Test, 2nd edition, DKEFS Delis-Kaplan Executive Function System.

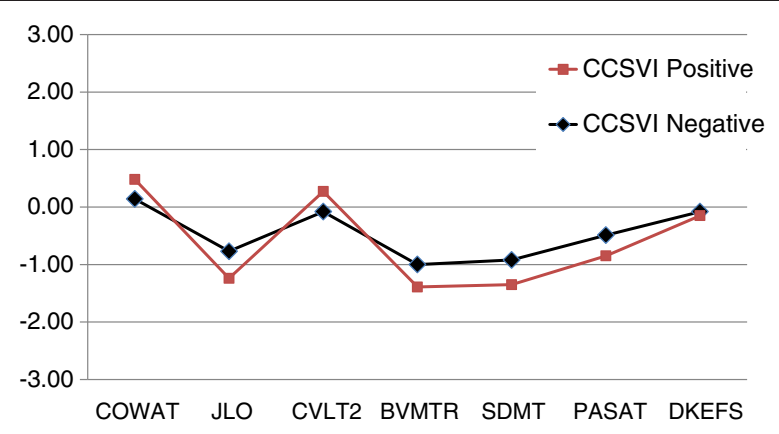

Figure 2 Cognitive profiles of chronic cerebrospinal venous insufficiency $(\mathrm{CCSVI})$ negative $(n=45)$ and positive $(n=64)$ multiple sclerosis patients. Each value represents a z score based on previously published normative data accounting for demographic variables. There are no significant differences for any test.

\section{Discussion}

To the best of our knowledge, this is the first investigation of neuropsychological status in MS patients in relation to CCSVI. In this sample of 109 MS patients, we find no evidence of an association between CCSVI and cognitive impairment. Moreover, no relationship between cognitive performance and the severity of CCSVI criteria, as determined by DS, was detected in linear regression analysis. When patients were categorized by their CCSVI status (positive/negative), significant group differences emerged for only one test, in a direction contrary to the hypothesis that CCSVI is a risk factor for cognitive impairment in MS. Similarly, there was no relationship between CCSVI and depression in this cohort.

The CCSVI hypothesis has provoked great controversy and debate in the MS research community since it was first presented $[11,13]$. The hypothesis gained popularity among MS patients because of the postulated possibility of venous insufficiency correction using endovascular procedures. While the diagnosis of CCSVI can be established using noninvasive and invasive imaging techniques [12], the validity of DS to establish the diagnosis of CCSVI remains controversial. We showed previously that DS, in properly trained hands, has high sensitivity and specificity for CCSVI diagnosis, when compared to invasive imaging methods $[48,49]$. This was the same method as used in this study, and thus we are confident in the validity of the CCSVI categorization in our analysis.

The true prevalence of CCSVI in MS patients is unknown, and there is good evidence that the condition is also found in patients with other neurologic diseases [23]. In this study, $64 \%$ of the participating MS subjects presented with CCSVI, which is similar to our previous study [23]. The difference between the prevalence rates of CCSVI-positive versus -negative MS patients in this study is modest, and of uncertain meaning with respect to MS pathology. Indeed, emerging studies point against 
CCSVI having a primary causative role in the development of MS [11,13]. A multimodal approach will likely be needed to determine the extent to which CCSVI is present in various healthy and disease groups and MS subtypes [15].

Cognitive impairment is common in MS and can be reliably quantified using neuropsychological tests emphasizing episodic memory, mental processing speed and some aspects of executive function [50]. Neuropsychological deficits are also robustly correlated with brain MRI measures, especially global and regional brain atrophy [51]. The heterogeneity of neuropsychological presentation among MS patients is influenced by many factors, including genetics, gender, intelligence, disease course, comorbid neuropsychiatric illness and health behaviors. The present study employed consensus standard tests emphasizing multiple domains of cognitive function, allowing us to test, in a comprehensive way, whether the presence and severity of CCSVI can influence this important sphere of disability in MS patients. No association between cognitive impairment and the presence and severity of CCSVI was found. This is consistent with our previous findings of a lack of association between the presence of CCSVI and severity of lesion burden and brain atrophy outcomes in MS patients [27].

There are a number of potential limitations in this study. Selection of participants was based on the inclusion or exclusion criteria in patients agreeing to undergo cognitive testing. However, it may be that the most severe patients presenting in our Center were not included in the study. Another potential limit is not including a control group. However, the aim of this study was not to assess CCSVI prevalence, but rather an association with cognitive impairment. Finally, the diagnosis of CCSVI was not confirmed by the use of other invasive diagnostic methods.

\section{Conclusions}

In conclusion, we find no evidence of an association between the presence and severity of CCSVI and cognitive impairment and depression in patients with MS.

\section{Abbreviations \\ CCSVI: Chronic cerebrospinal venous insufficiency; DS: Doppler sonography; IJV: Internal jugular vein.}

\section{Competing interests}

RHBB receives royalties from Psychological Assessment Resources that are in part associated with the Brief Visuospatial Memory Test Revised. RHBB has acted as a consultant or scientific advisory board member for Bayer, Biogen Idec, Actelion, and Novartis. He has received financial support for research activities from Shire Pharmaceuticals, Accorda and Biogen Idec. BW-G has participated in speaker's bureaus and served as a consultant for Biogen Idec, Teva Neurosciences, EMD Serono, Pfizer, Novartis, Genzyme, and Acorda. She also has received grant/research support from the agencies listed above as well as ITN, Questcor and Shire. No other industry financial relationships exist. DH has received speaker honoraria and consultant fees from Biogen Idec, Teva Pharmaceutical Industries Ltd., EMD Serono, Pfizer Inc, and Genzyme. RZ has received financial support for research activities from Biogen Idec, Teva Pharmaceutical and Teva Neuroscience, EMD Serono, Genzyme-Sanofi, Novartis, Greatbatch, Bracco and Questcor. He also received personal compensation from Teva Pharmaceutical, Biogen Idec, Novartis,
Genzyme-Sanofi, EMD Serono, Bayer, Novartis and General Electric for speaking and consultant services. KM, W, CK, EC and CB declare that they have no competing interests.

\section{Authors' contributions}

All authors participated in discussion and correspondence to develop this consensus opinion on the topics covered in this article. RHBB and RZ are the lead authors because they lead the project and wrote the first draft of the manuscript. All authors read and approved the final manuscript.

\section{Study disclosure}

The data used for this study were collected prospectively (between May 2010 and January 2011), as part of a self-referred, fee-for-service program in MS patients. Participants underwent clinical and imaging tests and were evaluated for the presence of CCSVI. All patients provided signed informed consent for entry of their test results in a central research database. The database registry included MRI of the brain and neck, Doppler examination of the brain and neck, neurological examination, neuropsychological testing and blood draw for genetic testing. The current study on cognition and CCSVI was funded by internal resources of the Buffalo Neuroimaging Analysis Center and Baird MS Center, the Jacobs Neurological Institute, University of Buffalo, as well as from the Direct MS Foundation and the Jacquemin Family Foundation.

\section{Author details}

'Department of Neurology, State University of New York at Buffalo, 100 High St, Buffalo, NY 14203, USA. ²Buffalo Neuroimaging Analysis Center, Department of Neurology, State University of New York at Buffalo, 100 High St, Buffalo, NY 14203, USA. ${ }^{3}$ Department of Neurology, School of Medicine and Biomedical Sciences, 100 High St, Buffalo, NY 14203, USA.

Received: 27 February 2013 Accepted: 17 June 2013

Published: 18 July 2013

\section{References}

1. Hauser SL, Oksenberg JR, Hauser SL, Oksenberg JR: The neurobiology of multiple sclerosis: genes, inflammation, and neurodegeneration. Neuron 2006, 52:61-76

2. Trapp BD, Nave KA: Multiple sclerosis: an immune or neurodegenerative disorder? Annu Rev Neurosci 2008, 31:247-269.

3. Rao SM, Leo GJ, Bernardin L, Unverzagt F: Cognitive dysfunction in multiple sclerosis. I. Frequency, patterns, and prediction. Neurology 1991, 41:685-691.

4. Benedict RHB, Cookfair D, Gavett R, Gunther M, Munschauer F, Garg N, Weinstock-Guttman B: Validity of the minimal assessment of cognitive function in multiple sclerosis (MACFIMS). J Int Neuropsychol Soc 2006, 12:549-558.

5. Patti F, Amato M, Trojano M, Bastianello S, Tola M, Goretti B, Caniatti L, Di Monte E, Ferrazza P, Brescia Morra V, Lo Fermo S, Picconi O, Luccichenti G, COGIMUS Study Group: Cognitive impairment and its relation with disease measures in mildly disabled patients with relapsing-remitting multiple sclerosis: baseline results from the Cognitive Impairment in Multiple Sclerosis (COGIMUS) study. Mult Scler 2009, 15:779-788.

6. Glanz BI, Holland CM, Gauthier SA, Amunwa EL, Liptak Z, Houtchens MK, Sperling RA, Khoury SJ, Guttmann CR, Weiner HL: Cognitive dysfunction in patients with clinically isolated syndromes or newly diagnosed multiple sclerosis. Mult Scler 2007, 13:1004-1010.

7. Houtchens MK, Benedict RHB, Killiany R, Sharma J, Jaisani Z, Singh B, Weinstock-Guttman B, Guttmann CRG, Bakshi R: Thalamic atrophy and cognition in multiple sclerosis. Neurology 2007, 69:113-123.

8. Roosendaal SD, Bendfeldt K, Vrenken H, Polman CH, Borgwardt S, Radue EW, Kappos L, Pelletier D, Hauser SL, Matthews PM, Barkhof F, Geurts JJ: Grey matter volume in a large cohort of MS patients: relation to MRI parameters and disability. Mult Scler 2011, 17:1098-1106.

9. Fisher E, Lee JC, Nakamura K, Rudick RA: Gray matter atrophy in multiple sclerosis: a longitudinal study. Ann Neurol 2008, 64:255-265.

10. Zamboni P, Galeotti R, Menegatti E, Malagoni AM, Tacconi G, Dall'Ara S, Bartolomei I, Salvi F: Chronic cerebrospinal venous insufficiency in patients with multiple sclerosis. J Neurol Neurosurg Psychiatry 2009, 80:392-399.

11. Khan O, Filippi M, Freedman MS, Barkhof F, Dore-Duffy P, Lassmann H, Trapp B, Bar-Or A, Zak I, Siegel MJ, Lisak R: Chronic cerebrospinal venous insufficiency and multiple sclerosis. Ann Neurol 2010, 67:286-290. 
12. Zivadinov R, Ramanathan M, Dolic K, Marr K, Karmon $Y$, Siddiqui AH, Benedict RH, Weinstock-Guttman B: Chronic cerebrospinal venous insufficiency in multiple sclerosis: diagnostic, pathogenetic, clinical and treatment perspectives. Expert Rev Neurother 2011, 11:1277-1294.

13. Rudick RA: Multiple sclerosis: is multiple sclerosis caused by venous insufficiency? Nat Rev Neurol 2010, 6:472-474

14. Zamboni P, Menegatti E, Galeotti R, Malagoni AM, Tacconi G, Dall'Ara S, Bartolomei I, Salvi F: The value of cerebral Doppler venous haemodynamics in the assessment of multiple sclerosis. J Neurol Sci 2009, 282:21-27.

15. Zivadinov R: Funding CCSVI research is/was a waste of valuable time, money and intellectual energy. No. Mult Scler 2013, 19:858-860.

16. Baracchini C, Perini P, Causin F, Calabrese M, Rinaldi F, Gallo P: Progressive multiple sclerosis is not associated with chronic cerebrospinal venous insufficiency. Neurology 2011, 77:844-850.

17. Batista S, Teter B, Sequeira K, Josyula S, Hoogs M, Ramanathan M, Benedict $\mathrm{RH}$, Weinstock-Guttman B: Cognitive impairment is associated with reduced bone mass in multiple sclerosis. Mult Scler 2012, 18:1459-1465.

18. Doepp F, Paul F, Valdueza JM, Schmierer K, Schreiber SJ: No cerebrocervical venous congestion in patients with multiple sclerosis. Ann Neurol 2010, 68:173-183.

19. Baracchini C, Perini P, Calabrese M, Causin F, Rinaldi F, Gallo P: No evidence of chronic cerebrospinal venous insufficiency at multiple sclerosis onset. Ann Neurol 2011, 69:90-99.

20. Centonze D, Floris R, Stefanini M, Rossi S, Fabiano S, Castelli M, Marziali S, Spinelli A, Motta C, Garaci FG, Bernardi G, Simonetti G: Proposed chronic cerebrospinal venous insufficiency criteria do not predict multiple sclerosis risk or severity. Ann Neurol 2011, 70:51-58.

21. Tsivgoulis G, Mantatzis M, Bogiatzi C, Vadikolias K, Voumvourakis K, Prassopoulos P, Piperidou C, Heliopoulos I: Extracranial venous hemodynamics in multiple sclerosis: a case-control study. Neurology 2011, 77:1241-1245.

22. Mayer CA, Pfeilschifter W, Lorenz MW, Nedelmann M, Bechmann I, Steinmetz H, Ziemann U: The perfect crime? CCSVI not leaving a trace in MS. J Neurol Neurosurg Psychiatry 2011, 82:436-440.

23. Zivadinov R, Marr K, Cutter G, Ramanathan M, Benedict RH, Kennedy C, Elfadil M, Yeh AE, Reuther J, Brooks C, Hunt K, Andrews M, Carl E, Dwyer MG, Hojnacki D, Weinstock-Guttman B: Prevalence, sensitivity, and specificity of chronic cerebrospinal venous insufficiency in MS. Neurology 2011, 77:138-144.

24. Weinstock-Guttman B, Ramanathan M, Marr K, Hojnack D, Benedict RH Morgan C, Yeh EA, Carl E, Kennedy C, Reuther J, Brooks C, Hunt K, Elfadil M, Andrews M, Zivadinov R: Clinical correlates of chronic cerebrospinal venous insufficiency in multiple sclerosis. BMC Neurol 2012, 12:26.

25. Dolic K, Marr K, Valnarov V, Dwyer MG, Carl E, Hagemeier J, Kennedy C, Brooks C, Kilanowski C, Hunt K, Hojnacki D, Weinstock-Guttman B, Zivadinov $R$ : Sensitivity and specificity for screening of chronic cerebrospinal venous insufficiency using a multimodal non-invasive imaging approach in patients with multiple sclerosis. Funct Neurol 2011, 26:205-214.

26. Bastianello S, Romani A, Viselner G, Tibaldi EC, Giugni E, Altieri M, Cecconi $P$, Mendozzi L, Farina M, Mariani D, Galassi A, Quattrini C, Mancini M, Bresciamorra V, Lagace A, McDonald S, Bono G, Bergamaschi R: Chronic cerebrospinal venous insufficiency in multiple sclerosis: clinical correlates from a multicentre study. BMC Neurol 2011, 11:132.

27. Zivadinov R, Cutter G, Marr K, Ramanathan M, Benedict RH, Bergsland N, Morgan C, Carl E, Hojnacki D, Yeh EA, Willis L, Cherneva M, Kennedy C, Dwyer MG, Weinstock-Guttman B: No association between conventional brain MR imaging and chronic cerebrospinal venous insufficiency in multiple sclerosis. AJNR Am J Neuroradiol 2012, 33:1913-1917.

28. Kurtzke JF: Rating neurologic impairment in multiple sclerosis: an expanded disability status scale (EDSS). Ann Neurol 1983, 13:227-231.

29. Lublin FD, Reingold SC: Defining the clinical course of multiple sclerosis: results of an international survey. National Multiple Sclerosis Society (USA) Advisory Committee on Clinical Trials of New Agents in Multiple Sclerosis. [see comment]. Neurology 1996, 46:907-911.

30. Beck AT, Steer RA, Brown GK: BDI-Fast Screen for Medical Patients: Manual. San Antonio, TX: Psychological Corporation; 2000

31. Benedict RHB, Fishman I, McClellan MM, Bakshi R, Weinstock-Guttman B: Validity of the beck depression inventory - fast screen in multiple sclerosis. Mult Scler 2003, 9:393-396.

32. Benedict RHB, Fischer JS, Archibald CJ, Arnett PA, Beatty WW, Bobholz J, Chelune G, Fisk JD, Langdon DW, Caruso LS, Foley F, LaRocca NG, Vowels L, Weinstein A, DeLuca J, Rao SM, Munschauer F: Minimal neuropsychological assessment of MS patients: a consensus approach. Clin Neuropsychol 2002, 16:381-397.
33. Parmenter BA, Testa SM, Schretlen DJ, Weinstock-Guttman B, Benedict RHB The utility of regression-based norms in interpreting the minimal assessment of cognitive function in multiple sclerosis (MACFIMS) J Int Neuropsychol Soc 2010, 16:6-16.

34. Benedict RHB: Effects of using same- versus alternate-form memory tests during short-interval repeated assessments in multiple sclerosis. $J$ Int Neuropsychol Soc 2005, 11:727-736.

35. Benedict R, Ramasamy D, Munschauer FE, Weinstock-Guttman B, Zivadinov $R$ : Memory impairment in multiple sclerosis: correlation with deep gray matter and mesial temporal atrophy. Mult Scler 2008, 14:S252.

36. Tekok-Kilic A, Benedict RHB, Weinstock-Guttman B, Dwyer MG, Carone D, Srinivasaraghavan B, Yella V, Abdelrahman N, Munschauer F, Bakshi R, Zivadinov R: Independent contributions of cortical gray matter atrophy and ventricle enlargement for predicting neuropsychological impairment in multiple sclerosis. Neurolmage 2007, 36:1294-1300.

37. Smith A: Symbol Digit Modalities Test: Manual. Los Angeles: Western Psychological Services; 1982.

38. Gronwall D: Paced auditory serial addition task: A measure of recovery from concussion. Percept Mot Skills 1977, 44:367-373.

39. Delis DC, Kramer JH, Kaplan E, Ober BA: Califorina Verbal Learning Test. 2nd edition. San Antonio, TX: The Psychological Corporation; 2000.

40. Benedict RHB, Schretlen D, Groninger L, Dobraski M, Shpritz B: Revision of the Brief Visuospatial Memory Test: studies of normal performance, reliability, and validity. Psychol Assess 1996, 8:145-153.

41. Benton AL, Hamsher KS: Multilingual Aphasia Examination. lowa City: IA: AJA Associates; 1989

42. Benton AL, Sivan AB, Hamsher K, Varney NR, Spreen O: Contributions to Neuropsychological Assessment. 2nd edition. New York: Oxford University Press; 1994.

43. Delis DC, Kaplan E, Kramer JH: Delis-Kaplan Executive Function System. San Antonio, TX: The Psychological Corporation; 2001.

44. Zamboni P, Menegatti E, Weinstock-Guttman B, Schirda C, Cox JL, Malagoni AM, Hojanacki D, Kennedy C, Carl E, Dwyer MG, Bergsland N, Galeotti R, Hussein S, Bartolomei I, Salvi F, Zivadinov R: The severity of chronic cerebrospinal venous insufficiency in patients with multiple sclerosis is related to altered cerebrospinal fluid dynamics. Funct Neurol 2009, 24:133-138.

45. Batista S, Zivadinov R, Hoogs M, Bergsland N, Heininen-Brown M, Dwyer MG, Weinstock-Guttman B, Benedict RH: Basal ganglia, thalamus and neocortical atrophy predicting slowed cognitive processing in multiple sclerosis. J Neurol 2012, 259:139-146.

46. Eshaghi A, Riyahi-Alam S, Roostaei T, Haeri G, Aghsaei A, Aidi MR, Pouretemad HR, Zarei M, Farhang S, Saeedi R, Nazeri A, Ganjgahi H, Etesam F, Azimi AR, Benedict RH, Sahraian MA: Validity and reliability of a Persian translation of the minimal assessment of cognitive function in multiple sclerosis (MACFIMS). Clin Neuropsychol 2012, 26:975-984.

47. Parmenter BA, Zivadinov R, Kerenyi L, Gavett R, Weinstock-Guttman B, Dwyer M, Garg N, Munschauer F, Benedict RHB: Validity of the Wisconsin card sorting and Delis-Kaplan executive function system (DKEFS) sorting tests in multiple sclerosis. J Clin Exp Neuropsychol 2007, 29:215-223.

48. Zivadinov R, Galeotti R, Hojnacki D, Menegatti E, Dwyer MG, Schirda C, Malagoni AM, Marr K, Kennedy C, Bartolomei I, Magnano C, Salvi F, Weinstock-Guttman B, Zamboni P: Value of MR venography for detection of internal jugular vein anomalies in multiple sclerosis: a pilot longitudinal study. AJNR Am J Neuroradiol 2011, 32:938-946.

49. Hojnacki D, Zamboni P, Lopez-Soriano A, Galleotti R, Menegatti E, WeinstockGuttman B, Schirda C, Magnano C, Malagoni AM, Kennedy C, Bartolomei I, Salvi F, Zivadinov R: Use of neck magnetic resonance venography, Doppler sonography and selective venography for diagnosis of chronic cerebrospina venous insufficiency: a pilot study in multiple sclerosis patients and healthy controls. Int Angiol 2010, 29:127-139.

50. Benedict RH, Zivadinov R: Risk factors for and management of cognitive dysfunction in multiple sclerosis. Nat Rev Neurol 2011, 7:332-342

51. Filippi M, Rocca MA, Benedict RH, DeLuca J, Geurts JJ, Rombouts SA, Ron M, Comi G: The contribution of MRI in assessing cognitive impairment in multiple sclerosis. Neurology 2010, 75:2121-2128.

doi:10.1186/1741-7015-11-167

Cite this article as: Benedict et al:: Chronic cerebrospinal venous insufficiency is not associated with cognitive impairment in multiple sclerosis. BMC Medicine 2013 11:167. 